\title{
ON THE LINEAR REPRESENTATION OF BRAID GROUPS
}

\author{
D. D. LONG
}

\begin{abstract}
We give a new derivative of the Burau and Gassner representations of the braid and pure braid groups. Various applications are explored.
\end{abstract}

\section{INTRODUCTION}

In [7], Jones announced his celebrated polynomial, using a construction based on the theory of Hecke and von Neumann algebras. One of the most interesting and mysterious aspects of this approach was the discovery of new representations of the braid groups, which generalized the classical Burau representation.

In this paper we shall give new and rather natural constructions for the classical Burau and Gassner representations; indeed the procedure will give representations for large classes of subgroups of the automorphism group of a free group. Our motivation for doing this is twofold; first, the Burau representation itself has been the object of intense study, in particular the question of its faithfulness has proved to be very elusive, at least in some part due to the difficulties involved in its construction. Our approach will be to use representation varieties to obtain the groups as groups of diffeomorphisms and then make the representations amenable to methods of differential geometry and the theory of Lie groups. Second, an easy generalization of these constructions will give rise to certain summands in the Jones representations of the braid groups.

The organization of the paper is as follows. In $\S 2$ we show how to obtain a faithful representation of $\operatorname{Aut}\left(F_{n}\right)$ as a group of diffeomorphisms. This is the basis of our construction of linear representations and proceeds by using the natural action of $\operatorname{Aut}\left(F_{n}\right)$ on a representation variety $R=R\left(F_{n}, G\right)$, where $G$ is any compact semisimple Lie group. Then by exact analogy with the construction of the Magnus representations [2], we restrict to a subgroup $H$ and observe that if there are global fixed points for $H$ inside $R$, then we have an obvious way to linearize; namely, the derivative.

In special cases there are large numbers of fixed points, and this enables us to construct linear representations:

Received by the editors September 20, 1987.

1980 Mathematics Subject Classification (1985 Revision). Primary 20F36; Secondary 20F29, 20F34, 57M05.

Partially supported by NSF Grant No. DMS-8701422. 
Theorem. Suppose that $\alpha$ is a $k$-cube of representations fixed by every element of $H$. Then we obtain a $k$-parameter family of linear representations of $H$ by $H \rightarrow \mathrm{GL}\left(T_{\alpha} R\right)$.

This is of interest even in special cases, for example a calculation shows:

Theorem. In the case that $G=\mathrm{SU}(2, \mathbb{C})$ we have

(a) If $H=B_{n}$, the $n$-strand braid group, then there is a circle $\alpha$ of fixed representations, and the representation $\rho_{t}: B_{n} \rightarrow \mathrm{GL}\left(T_{\alpha(t)} R\right)$ splits as the obvious permutational representation together with the Burau representation.

(b) If $H=P_{n}$, the $n$-strand pure braid group, then there is an $n$-torus $\beta$ of fixed representations and there is an n-parameter representation

$$
g_{\beta}: P_{n} \rightarrow \mathrm{GL}\left(T_{\beta} R\right)
$$

which splits as a (trivial) permutational representation together with the Gassner representation.

This should be contrasted with the usual ways of obtaining these representations-either using the free differential calculus or by considering the homology of a certain covering as a module over the group ring of the covering group. Both of these approaches seem very hard to work with and it is to be hoped that this approach may provide some new insights and bring to bear other methods on the important open question of whether these representations are faithful. This result could be interpreted as an answer to question 22 of [2]; namely a "natural" interpretation of the Burau and Gassner matrices.

In fact, further generalizations are possible by considering the spaces of differential $p$-tensors $\mathscr{T}^{p}$ over $R$. We have by analogy:

Theorem. With the notation established above, there is a k-parameter family of representations of $H$ given by $H \rightarrow \mathrm{GL}\left(\mathscr{T}^{p}(R)_{\alpha}\right)$.

We can further specialize to the case of alternating $p$-forms; it turns out here that there is a natural invariant subspace on which any representation of the braid group is "quadratic" in the sense of Jones so must come from the Hecke algebra. In this way we are able to show:

Theorem. The representation of $B_{n}$ coming from the above theorem contains an irreducible summand of the Jones representation with the Young diagram $\left(n-p, 1^{p}\right)$.

This result was essentially first proved in [8]. Additional structure is also available since the diffeomorphisms appearing in the image are far from arbitrary; we show for example that they have an "analytic" type of property of being determined by their action on any open set.

The group structure gives further restrictions, for example the representation variety can be foliated in a way which is invariant for the group. We also show the existence of invariant symmetric 2-tensors, first observed in [15]. This comes from writing down a height function which is clearly invariant for braid group 
diffeomorphisms. When restricted to the leaves of the foliation, this height function has a critical point, and hence a Hessian. As above, we can consider a path of Hessians along $\alpha$. In this way we show:

Theorem. (a) The reduced Burau representation acts as a group of isometries of a certain nondegenerate pairing on a subspace of $T_{\alpha}(R)$.

(b) The reduced Gassner representation acts as a group of isometries of a certain nondegenerate pairing on a subspace of $T_{\beta}(R)$.

In particular, in the first case one obtains a one-parameter family of symmetric matrices, which essentially describes the form of [15]; in the second case, one obtains an $n$-parameter family of symmetric matrices. The word "nondegenerate" here is interpreted as "generically nondegenerate".

In $\S 4$ we give some examples and describe other potential applications of these ideas.

I thank J. D. Moore and S. P. Humphries for enlightening conversations, and the referee and J. Kidder for some useful comments.

\section{REPRESENTATION BY DIFFEOMORPHISMS}

Throughout, we shall fix a compact semisimple Lie group $G$ which for almost all of our purposes can be considered to be $\operatorname{SU}(2, \mathbb{C})$. Let $F_{n}$ be the free group of rank $n$. Set $R=R\left(F_{n}, G\right)$, the representation variety of $F_{n}$, topologized by the compact open topology.

Fix once and for all some generating set $x_{1}, x_{2}, \ldots, x_{n}$ for $F_{n}$. Using this basis we see that since the group is free, any $n$-tuple of matrices determine a representation, and conversely, any representation determines an $n$-tuple of matrices. In this way we may identify the representation space $R$ with $G^{n}=$ $G \times \cdots \times G$.

Suppose now that we are given an automorphism $\theta$ of the free group. Then this acts on the representation space by $\rho \rightarrow \theta^{*} \rho$, where the latter representation is defined by the rule

$$
\theta^{*} \rho(w)=\rho\left(\theta^{-1}(w)\right), \quad w \in F_{n} .
$$

The reason for the inversion will soon become apparent. This action appears as the map $\theta^{*}: G^{n} \rightarrow G^{n}$ given by

$$
\theta^{*}:\left(X_{1}, \ldots, X_{n}\right) \rightarrow\left(\theta_{*}^{-1} X_{1}, \ldots, \theta_{*}^{-1} X_{n}\right),
$$

where this notation is to be interpreted in the obvious way. For example, if $\theta$ is the automorphism of the free group of rank two defined by $\theta(x)=x \cdot y \cdot x$, $\theta(y)=x \cdot y$, then the map $\theta^{*}$ has action $(X, Y) \rightarrow\left(Y^{-1} \cdot X, X^{-1} \cdot Y^{2}\right)$. The map $\theta^{*}$ is said to be the map associated to $\theta$. Observe that the map $\theta^{*}$ is not a Lie group homomorphism; that is $\theta^{*}\left(\rho_{1} \rho_{2}\right) \neq \theta^{*}\left(\rho_{1}\right) \theta^{*}\left(\rho_{2}\right)$. However, since $\theta$ is an automorphism, $\theta^{*}$ is clearly a diffeomorphism.

Notice that with the given definition, the map $\operatorname{Aut}\left(F_{n}\right) \rightarrow \operatorname{Diff}(R)$ given by $\theta \rightarrow \theta^{*}$ is a group homomorphism; it is at this stage that the inversion is necessary, otherwise the map would be an antihomomorphism. 
Moreover, one of the pleasant features about this construction is the following rather easy consequence.

Theorem 2.1. The representation $\operatorname{Aut}\left(F_{n}\right) \rightarrow \operatorname{Diff}(R)$ is faithful.

Proof. A result of Epstein [5] implies that we may choose an $n$-tuple of matrices $\left(X_{1}, X_{2}, \ldots, X_{n}\right)$ in $G$, so that the group generated by $\left\{X_{1}, X_{2}, \ldots, X_{n}\right\}$ is a free group on $n$ generators. (In fact he proves that this situation is generic in the sense that if one uses Haar measure on $G^{n}$ then the set of $n$-tuples for which this is not true has measure zero.)

It follows that if $\theta$ is not the identity automorphism, without loss of generality, we may assume that $x_{1}$ is different from $\theta\left(x_{1}\right)$. Consider the representation $\rho$ of the free group given by $\rho\left(x_{i}\right)=X_{i}$. Since with the given choices this representation is faithful, the elements $X_{1}$ and $\theta^{*}\left(X_{1}\right)$ are different, so that the associated map moves $\rho$ and is not the identity on $R$.

Remark. This construction has also been exploited in different contexts; see [10]. It is also implicit from results in [11] that if $\sigma \in B_{n}$ is a noncentral element, then for generic $X$ and $Y, \operatorname{trace}\left(\sigma^{*}(X Y)\right) \neq \operatorname{trace}(X Y)$. See also the proof of 4.7(b).

Another corollary of the way that these maps were constructed is that they have the following "analytic" property.

Corollary 2.2. Let $\theta^{*}$ be an automorphism in the image of $B_{n}$. Then $\theta$ is determined by its restriction to any open set in $R$.

Proof. By [5], such an open set contains a representation $\rho$ which is a free group; whence the automorphism $\theta$ can be reconstructed from the action of $\theta^{*}$ on $\rho$. This then determines $\theta^{*}$ everywhere on $R$.

We now proceed to use the methods of smooth topology to construct linear representations of certain subgroups of $\operatorname{Aut}\left(F_{n}\right)$. This will be closely related to the Magnus construction of [2]. We shall proceed first in a slightly special case and then generalize.

Suppose then that $H$ is some subgroup of $\operatorname{Aut}\left(F_{n}\right)$ which we seek to represent. Assume that there is a path of representations $\alpha:(-1,1) \rightarrow R$ which have the property that for every $h \in H, h^{*} \alpha(t)=\alpha(t)$. (For example, in the cases below, we could take $H$ to be the subgroup of $\operatorname{Aut}\left(F_{n}\right)$ which acts as the identity on the homology of $F_{n}$; then $\alpha$ is any path lying inside the abelian representations.)

Then we have

Theorem 2.3. There is a one-parameter family of representations of $H$, defined by the composition

$$
\rho_{t}: H \rightarrow \operatorname{Diff}(R) \rightarrow \mathrm{GL}\left(T_{\alpha(t)}(R)\right),
$$

where $\rho_{t}$ is defined by the rule $\rho_{t}(h)=d h_{\alpha(t)}^{*}$.

Proof. There is almost nothing to check; the chain rule guarantees that this is a representation. 
We shall show by example that this gives rise to highly nontrivial representations. Note also that the restriction that $\alpha$ be a path is not necessary; in general we can choose any $k$-cube; this will give representations with $k$-parameters.

We now give some examples of Theorem 2.3. Our main interest here will be in the braid group and the pure braid group; full information about these groups and their main properties can be found in [2]. We recall briefly the salient details.

The $n$-string braid group $B_{n}$ we shall define to be the subgroup of $\operatorname{Aut}\left(F_{n}\right)$ generated by the automorphisms $\left\{\sigma_{i} \mid 1 \leq i \leq n-1\right\}$ where the action of $\sigma_{i}$ is given by

$$
\begin{aligned}
& x_{i} \rightarrow x_{i+1}, \\
& x_{i+1} \rightarrow\left(x_{i+1}\right)^{-1} \cdot x_{i} \cdot x_{i+1}, \\
& x_{j} \rightarrow x_{j}, \quad j \neq i, i+1 .
\end{aligned}
$$

This group arises in nature in many ways, in particular as the group of automorphisms of the disc with $n$ puncture points, where two automorphisms are regarded as equivalent if they are isotopic via an isotopy fixed on the points and the boundary. However we shall have no need to refer to this in this paper.

If we use the symbol $\Sigma_{n}$ to denote the symmetric group on $n$ letters, then there is an obvious map from $B_{n} \rightarrow \Sigma_{n}$ coming from action as a permutation group on the set $\left\{x_{1}, \ldots, x_{n}\right\}$. We now define the pure braid group, denoted by $P_{n}$, to be the kernel of this homomorphism.

Two facts about these groups which we shall need to refer to later, and which are obvious given the above description are the following:

(i) If $\theta \in B_{n}\left(\right.$ or $\left.P_{n}\right)$ then $\theta\left(x_{1} \cdots x_{n}\right)=x_{1} \cdots x_{n}$.

(ii) If $\left(X_{1}, \ldots, X_{n}\right)$ is a representation and $\theta \in B_{n}$, then $\theta^{*}$ acts as a permutation of the elements $\left\{\operatorname{tr}\left(X_{1}\right), \ldots, \operatorname{tr}\left(X_{n}\right)\right\}$. Indeed if $\theta^{*}$ lies in $P_{n}$, then this permutation is trivial.

Since we are interested in doing explicit calculations, we also collect some rudimentary facts about $\mathrm{SU}(2, \mathbb{C})$ and its Lie algebra $\mathfrak{S}$.

Recall that $\operatorname{SU}(2, \mathbb{C})$ is the group of unitary matrices of determinant 1 , so that the general element has the form $\left[\begin{array}{cc}a & b \\ -b & a\end{array}\right]$ where the overbar denotes complex conjugation.

Let $\mathfrak{S}$ be the Lie algebra of $\operatorname{SU}(2, \mathbb{C})$ that is the tangent space at the identity. Then we may consider the tangent space at an arbitrary point $p \in \operatorname{SU}(2, \mathbb{C})$ as the Lie algebra $\mathfrak{S}$ translated to $p$ by multiplication on the right.

Further, a basis for $\mathfrak{S}$ is given by one-parameter subgroups running through the identity. That is, smooth maps $v:(-\varepsilon, \varepsilon) \rightarrow \mathrm{SU}(2, \mathbb{C}), v(0)=\mathrm{Id}$, where the tangent vector associated to the map $v(t)$ is the derivative $\frac{d}{d t} v(t)$ evaluated at $t=0$. To avoid cumbersome notation, we shall also use the symbol $\mathbf{v}$ for the vector defined by $\left.\frac{d}{d t} v(t)\right|_{t=0}$. It is now a matter of easy calculation to verify that we can identify $\mathfrak{S}$ with matrices

$$
\left[\begin{array}{cc}
i s & 0 \\
0 & -i s
\end{array}\right], \quad s \in \mathbf{R}, \quad \text { and } \quad\left[\begin{array}{cc}
0 & \xi \\
-\bar{\xi} & 0
\end{array}\right], \quad \xi \in \mathbb{C} \text {. }
$$


Vectors of the first type we shall call real and those of the second complex. (We shall have no need for the Lie algebra structure here, so we shall suppress reference to it.)

In order to compute the action of a diffeomorphism on the tangent space at a point $p$, we proceed as follows. Let $v(t) \cdot p$ be a smooth path running through the point $p$ and defining the tangent vector $\left.\frac{d}{d t} v_{t}\right|_{t=0} \cdot p=\mathbf{v} \cdot p$. Then we consider the composition $f(v(t) \cdot p)$; this is a path running through $f(p)$, and its derivative at zero is the vector $d f_{p}(\mathbf{v})$.

We shall also have cause to refer to the adjoint action of $\mathrm{SU}(2, \mathbb{C})$. If $M \in$ $\mathrm{SU}(2, \mathbb{C})$, then we define a diffeomorphism $\mathrm{SU}(2, \mathbb{C}) \rightarrow \mathrm{SU}(2, \mathbb{C})$ by

$$
X \rightarrow M \cdot X \cdot M^{-1} \text {. }
$$

This diffeomorphism is denoted $\operatorname{Ad}(M)$, and since is fixes the identity, it defines a map $\mathrm{SU}(2, \mathbb{C}) \rightarrow \operatorname{Aut}(\mathfrak{S})$.

Throughout this paper we shall adapt the notation that the symbol $\Lambda$ is the $\mathrm{SU}(2, \mathbb{C})$ matrix $\left[\begin{array}{ll}\lambda & 0 \\ 0 & \lambda\end{array}\right]$, where $\lambda$ is a complex number of modulus 1 . In certain special cases, we will wish to be more specific; $\Lambda_{p}$ will be the diagonal matrix with $\lambda=e^{i p}$.

This concludes our preliminary remarks and we now return to the matter in hand. Let $\alpha(p)$ be the curve running in $R$ through the points $\left(\Lambda_{p}, \ldots, \Lambda_{p}\right)$ for $0 \leq p \leq 2 \pi$. The action of a generator of $B_{n}$ on the free group consists of a permutation and a conjugacy, so that each representation along $\alpha(p)$ is fixed by the entire action of $B_{n}$. We may therefore apply the theorem to obtain linear representations of $B_{n}$. We now identify these representations. To state the result here, recall that there is a well-known representation of the braid group; the Burau representation (see $[3,4]$ ).

Theorem 2.4. The map $B_{n} \rightarrow \operatorname{Diff}\left(\mathrm{SU}(2, \mathbb{C})^{n}\right) \rightarrow \operatorname{GL}\left(T_{\alpha(p)}\left(\mathrm{SU}(2, \mathbb{C})^{n}\right)\right)$ yields a linear representation which splits as a permutational representation, together with a copy of the Burau representation.

Remark. The parameter $t$ of the Burau representation appears here as $\lambda^{2}$, so that although we have allowed $p$ to very in $[0,2 \pi]$ all representations are obtaned in $[0, \pi]$.

Proof. By the chain rule, it suffices to check the generators; and the map $\sigma_{1}$ is sufficently representative. With our conventions, we should work with the automorphism $\sigma_{1}^{-1}$; this is the map of the free group

$$
\begin{aligned}
& x_{1} \rightarrow x_{1} \cdot x_{2} \cdot x_{1}^{-1}, \\
& x_{2} \rightarrow x_{1}, \\
& x_{i} \rightarrow x_{i}, \quad i \geq 2 .
\end{aligned}
$$

It is an elementary fact that $T_{\alpha}(R)=T_{\alpha(p)}\left(\mathrm{SU}(2, \mathbb{C})^{n}\right)$ splits as the direct sum

$$
T_{\alpha(p)}(\mathrm{SU}(2, \mathbb{C})) \times T_{\alpha(p)}(\mathrm{SU}(2, \mathbb{C})) \times \cdots \times T_{\alpha(p)}(\mathrm{SU}(2, \mathbb{C})) .
$$


Clearly the map associated to $\sigma_{1}$ acts as the identity on the last $n-2$ factors. We can now proceed with our calculation. Consider the first component vectors in $R$. In our case, we may think of such a tangent vector to $\left(\Lambda_{p}, \Lambda_{p}, \ldots\right.$, $\left.\Lambda_{p}\right)$ as a path $\left(v_{t} \Lambda_{p}, \Lambda_{p}, \ldots, \Lambda_{p}\right)$, where $v_{0}=$ Id and the tangent vector in $\mathfrak{S}$ is $\mathbf{v}=\left.\frac{d}{d t} v_{t}\right|_{t=0}$. The associated map of $\sigma_{1}$ has action

$$
\left(v_{t} \Lambda_{p}, \Lambda_{p}, \ldots, \Lambda_{p}\right) \rightarrow\left(\left(v_{t} \Lambda_{p}\right) \cdot \Lambda_{p} \cdot\left(v_{t} \Lambda_{p}\right)^{-1}, v_{t} \Lambda_{p}, \Lambda_{p}, \ldots, \Lambda_{p}\right) .
$$

The image can be rewritten

$$
\left(v_{t} \cdot \Lambda_{p} \cdot v_{t}^{-1}, v_{t} \Lambda_{p}, \Lambda_{p}, \ldots, \Lambda_{p}\right)=\left(\left(v_{t} \cdot \Lambda_{p} \cdot v_{t}^{-1} \Lambda_{p}^{-1}\right) \Lambda_{p}, v_{t} \Lambda_{p}, \Lambda_{p}, \ldots, \Lambda_{p}\right)
$$

where the right-hand side is so written to emphasize the fact that the vector is regarded as based at the point $\left(\Lambda_{p}, \ldots, \Lambda_{p}\right)$. When differentiating in the first factor, we must use the product rule, which yields

$$
\left.\left.\left(\frac{d}{d t} v_{t}\right) \cdot \Lambda_{p} \cdot v_{t}^{-1} \Lambda_{p}^{-1}\right) \Lambda_{p}+v_{t} \cdot \Lambda_{p} \cdot \frac{d}{d t}\left(v_{t}^{-1}\right) \cdot \Lambda_{p}^{-1}\right) \Lambda_{p} .
$$

Now evaluating at $t=0$, we see that in the first component we now have the vector $\mathbf{v}-\Lambda_{p} \cdot \mathbf{v} \cdot\left(\Lambda_{p}\right)^{-1}$. A similar computation in the second coordinate gives the derivative as the map

$$
(\mathbf{v}, 0,0,0, \ldots, 0) \rightarrow\left(\mathbf{v}-\Lambda_{p} \cdot \mathbf{v} \cdot\left(\Lambda_{p}\right)^{-1}, \mathbf{v}, 0,0, \ldots, 0\right) .
$$

In a similar way, we see that a tangent vector $\left(\Lambda_{p}, w_{t} \Lambda_{p}, \ldots, \Lambda_{p}\right)$ is mapped:

$$
\left(\Lambda_{p}, w_{t} \Lambda_{p}, \ldots, \Lambda_{p}\right) \rightarrow\left(\Lambda_{p} \cdot w_{t} \Lambda_{p} \cdot\left(\Lambda_{p}\right)^{-1}, \Lambda_{p}, \ldots, \Lambda_{p}\right)
$$

This yields the map

$$
(0, \mathbf{w}, 0, \ldots, 0) \rightarrow\left(\Lambda_{p} \cdot \mathbf{w} \cdot \Lambda_{p}^{-1}, 0,0, \ldots, 0\right) .
$$

An easy calculation shows that the action of $\operatorname{Ad}\left(\Lambda_{p}\right)$ on the real part of $\mathfrak{S}$ is trivial, and so the real parts of each factor of $\bigoplus T_{\alpha(p)}(\mathrm{SU}(2, \mathbb{C}))$ form a real $n$-dimensional invariant subspace. On this subspace the representation of $\sigma_{1}$ becomes permutational:

$$
\left[\begin{array}{cccccc}
0 & 1 & 0 & \ldots & \ldots & 0 \\
1 & 0 & 0 & \ldots & \ldots & 0 \\
0 & 0 & 1 & 0 & \ldots & 0 \\
0 & 0 & 0 & 1 & \ldots & 0 \\
\ldots & \ldots & \ldots & \ldots & \ldots & \ldots \\
0 & 0 & 0 & \ldots & \ldots & 1
\end{array}\right]
$$

The complex pieces in each factor also form an invariant subspace; this being $n$-complex dimensional. Here the action of $\operatorname{Ad}\left(\Lambda_{p}\right)$ is multiplication by $\lambda^{2}$; whence the representation of $\sigma_{1}$ becomes

$$
\left[\begin{array}{cccccc}
1-\lambda^{2} & \lambda^{2} & 0 & \ldots & \ldots & 0 \\
1 & 0 & 0 & \ldots & \ldots & 0 \\
0 & 0 & 1 & 0 & \ldots & 0 \\
0 & 0 & 0 & 1 & \ldots & 0 \\
\ldots & \ldots & \ldots & \ldots & \ldots & \ldots \\
0 & 0 & 0 & \ldots & \ldots & 1
\end{array}\right]
$$


This is the unreduced Burau matrix for $\sigma_{1}$, with $t=\lambda^{2}$.

Remarks. (a) This construction was forced to give a reducible representation, since the path $\alpha(t)$ through $\alpha\left(t_{0}\right)$ defines a 1-eigenvector for all of the $B_{n}$ action on $T_{\alpha\left(t_{0}\right)}\left(\mathrm{SU}(2, \mathbb{C})^{n}\right)$.

(b) The appearance of $\lambda^{2}$ as a parameter is suggestive; in many recent results on linear representation of the braid group, there are occasions where the substitution $s^{2}=t$ was made in the Burau representation. (See [15], and the original von Neumann algebra approach, for example.)

In this way we see that one can interpret the faithfulness of the Burau representation as a condition on the diffeomorphisms that occur in the image of the map $B_{n} \rightarrow \operatorname{Diff}\left(\operatorname{SU}(2, \mathbb{C})^{n}\right)$; the Taylor series across the arc $\alpha$ cannot always be the identity to first order. Notice that by the Epstein lemma, nothing is lost in passing to the group of diffeomorphisms, so that a nonidentity element of $B_{n}$ gives rise to a nontrivial diffeomorphism; and indeed the same result shows that points are moved arbitrarily close to the $\alpha$-path. The issue is whether they are moved to first order. Any such proof of faithfulness must also use the fact that the diffeomorphism lies in $B_{n}$; there are diffeomorphisms which lie in the image of $\operatorname{Aut}\left(F_{n}\right)$ which fix the whole circle and whose derivative is the identity all along the circle.

There is an analogue for the pure braid group constructed in much the same way. We consider the $n$-torus $\beta\left(i_{0}, i_{1}, \ldots, i_{n}\right)=\left(\Lambda_{i_{1}}, \Lambda_{i_{2}}, \ldots, \Lambda_{i_{n}}\right)$, where each of the parameters lies in the interval $[0,2 \pi]$. Again, this $n$-torus is fixed by the action of the whole pure braid group and we can perform a similar analysis to linearize:

Theorem 2.5. The map $P_{n} \rightarrow \operatorname{Diff}\left(\mathrm{SU}(2, \mathbb{C})^{n} \rightarrow \mathrm{GL}\left(T_{\beta}\left(\mathrm{SU}(2, \mathbb{C})^{n}\right)\right)\right.$ yields a linear representation which splits as a (trivial) permutational representation, together with a copy of the Gassner representation.

Proof. This is an identical calculation to that of Theorem 2.4.

Remarks. (a) Another class of groups which can be represented in this fashion is the so-called $I A(n)$ automorphism group. This is the kernel of the map $\operatorname{Aut}\left(F_{n}\right) \rightarrow \operatorname{Aut}\left(F_{n} /\left[F_{n}, F_{n}\right]\right)$. Exactly as above, this group preserves the same $n$-torus as that of the Gassner representation. This yields a $n$-parameter linear representation for $I A(n)$.

(b) Since the representation of $F_{n}$ which maps everything to Id is clearly fixed by all of $\operatorname{Aut}\left(F_{n}\right)$, we obtain a representation of the whole group by linearizing here; it is easy to compute that this is a highly nontrivial representation, namely the natural representation obtained by abelianization of $F_{n}$.

At this state it is natural to ask to what extent the representations depend on the choices involved; in particular, the choice of path and Lie group. We now interject a short digression dealing wih these issues.

It is easy to see that whatever semisimple $G$ was chosen, the only possibility for representation invariant under the wholde braid group are of the form 
$(M, \ldots, M)$, and the only possibilities for the pure braid group are abelian representations. In either case, the representation will lie in some maximal torus for $G$.

The adjoint action of the maximal torus on the Lie algebra of $G$ is well understood; it splits as a sum of trivial one-dimensional representations (which come from the Lie algebra of the maximal torus), and two-dimensional rotational representations (which give rise to the roots of $G$ ). This is basically a consequence of the fact that over an algebraically closed field, Schur's lemma gives that the irreducible representations of an abelian group are one-dimensional; see $[1, \S 4.10$ et fol], for a very elementary account.

In our case we disguised the 2-dimensional representations by considering them as 1 -complex dimensional representations, where the rotation is given by a complex number of modulus 1 . So our choices represent no essential loss.

There is a further construction which we can perform which generalizes this construction. To describe it we need to recall some elementary facts from the theory of differential forms.

Let $V$ be a finite-dimensional vector space over a field $K$, say with zero characteristic. Then a $p$-tensor is a multilinear map $\omega: V \times \cdots \times V \rightarrow K$. As usual, all such maps can be considered as linear maps from the $K$ vector space $V \otimes \cdots \otimes V$ down to the ground field, that is, an element of the dual space of $V \otimes \cdots \otimes V$.

Define the bundle $T^{\otimes p} M$ to be the bundle whose fiber over a point $x$ is the vector space dual to $T_{x}(M) \otimes \cdots \otimes T_{x}(M)$. Such a bundle exists; see for example [12 or 14]. Then a $p$-form on $M$ is a smooth map $\omega: M \rightarrow T^{\otimes p} M$, so that for every $x$, the element $\omega(x)$ is in the dual space of $T_{x}(M) \otimes \cdots \otimes T_{x}(M)$. The space of $p$-forms can be made into a $K$ vector space in the obvious way, and we denote it $\mathscr{T}^{p}(M)$. Similarly, the $p$-forms at a point $x$ will be denoted by $\mathscr{T}^{p}(M)_{x}$.

If $f: M \rightarrow N$ is a smooth map, there is an induced map $f^{*}: \mathscr{T}^{p}(N) \rightarrow$ $\mathscr{T}^{p}(M)$ defined in the obvious way; given a $p$-form $\omega$ on $N$, this defines for each $y \in N$ a map $\omega(y): T_{y}(N) \otimes \cdots \otimes T_{y}(N) \rightarrow \mathbf{R}$. Then we define the form $f^{*} \omega$ by the rule that $f^{*} \omega(x)$ is the map defined by the composition

$$
d f_{x} \otimes \cdots \otimes d f_{x}: T_{x}(M) \otimes \cdots \otimes T_{x}(M) \rightarrow T_{f(x)}(N) \otimes \cdots \otimes T_{f(x)}(N) \rightarrow \mathbf{R} .
$$

Of course this definition is contravariant, so that $(g \circ f)^{*}=f^{*} \circ g^{*}$, which is bad from the group theoretic point of view. However in our case, the maps on the representation variety are diffeomorphisms, so that there is an induced map on $\mathscr{T}^{p}(R), \mathscr{T} f: \mathscr{T}^{p}(R) \rightarrow \mathscr{T}^{p}(R)$, which we define by $\mathscr{T} f=\left(f^{-1}\right)^{*}$. This is now a group homomorphism.

Since no confusion is possible, we suppress the rank of the form in our notation for $f$. Then we have by exact analogy with Theorem 2.3

Theorem 2.6. Let $H \leq \operatorname{Aut}\left(F_{n}\right)$ be given. Suppose that $\alpha: I^{k} \rightarrow R$ is a smooth family of representations such that $h^{*}$ fixes all the representations in 
the image of $\alpha$. Then there is a $k$-parameter family of linear representations $H \rightarrow \operatorname{GL}\left(\mathscr{T}^{p}(R)_{\alpha}\right)$ given by $\theta \rightarrow \mathscr{T} \theta^{*}$.

Remark. In the case that $M$ is a Riemannian manifold, there is a canonical identification between tangent and cotangent spaces (or use the symplectic structure described in $\S 3$ ) so that this is a generalization of Theorem 2.3 , the latter being the case $p=1$. In particular, we have at least the potential to generate new linear representations of both the braid and pure braid groups.

Usually one makes a further decomposition of $\mathscr{T}^{p}(M)$ as $S^{p}(M) \oplus \Lambda^{p}(M)$, where $S^{p}(M)$ denotes the symmetric tensors and $\Lambda^{p}(M)$ denotes the skew symmetric tensors; this being an invariant decomposition. Thus by restriction we obtain subrepresentations. It is these which we now analyze.

Recall that following Jones [8], we define the Hecke algebra $H(n, t)$ to be the complex algebra defined by the presentation

$$
\left\langle g_{1}, \ldots, g_{n}\left|g_{i} g_{j}=g_{j} g_{i},\right| i-j \mid>1, g_{i} g_{i+1} g_{i}=g_{i+1} g_{i} g_{i+1}, g_{i}^{2}=(1-t) g_{i}+t\right\rangle
$$

Here $t$ is any complex number. The crucial fact defining the Hecke algebra is that there is a quadratic relation

$$
g^{2}=(1-t) g+t
$$

for each generator $g$; in other words, the group algebra of the braid group is linearized in one of the simplest ways possible.

In our case, we split the Lie algebra $\mathfrak{S}$ of $\mathrm{SU}(2, \mathbb{C})$ into a "real" subspace and a "complex" subspace, this decomposition being invariant for the action of the braid group. This gives a natural invariant subspace of the alternating $p$-forms, namely, those of the form $v_{i_{1}} \wedge v_{i_{2}} \wedge \cdots \wedge v_{i_{n}}$, where each $v_{k}$ is a complex vector. We denote this space by $\mathbb{C} \Lambda^{p}(M)$. Let us now observe that property $(*)$ is preserved on this subspace in the case of the braid group.

Theorem 2.7. The representation of the braid group $\rho_{t}: B_{n} \rightarrow \operatorname{GL}\left(\mathbb{C} \Lambda^{p}(M)_{\alpha(t)}\right)$ satisfies the relation (*).

Proof. Recall that if $\left\{v_{1}, \ldots, v_{n}\right\}$ is a basis for the vector space $V$, then a basis for $\Lambda^{p}(V)$ is given by objects of the form $v_{i_{1}} \wedge v_{i_{2}} \wedge \cdots \wedge v_{i_{n}}$ where $1 \leq i_{1}<i_{2}<\cdots<i_{n} \leq p$. Moreover since it suffices to check the result on the generators of the braid group, and these only move two vectors, we need only consider the case $p=2$.

Consider the case of the generator $\sigma_{1}$. In this case we need only verify the result in the cases $v_{1} \wedge v_{2}, v_{1} \wedge v_{j}, j \geq 3$, and $v_{2} \wedge v_{3}$. This is now a question of easy verification; we do the first case (the only really nontrivial one) as a sample. The result of Theorem 2.4 gives the unreduced Burau representation, so we use this action on the algebra. To cut down notation somewhat we replace 
$\lambda^{2}$ by the more traditional $t$ :

$$
\begin{aligned}
\sigma_{1}^{2}\left(v_{1} \wedge v_{2}\right) & =\left(\left(1-t+t^{2}\right) v_{1}+(1-t) v_{2}\right) \wedge\left(\left(t-t^{2}\right) v_{1}+t v_{2}\right) \\
& =\left(-(1-t)\left(t-t^{2}\right)+\left(1-t+t^{2}\right) \cdot t\right) v_{1} \wedge v_{2}=t^{2} v_{1} \wedge v_{2}, \\
(1-t) \sigma_{1}\left(v_{1} \wedge v_{2}\right) & \left.=(1-t) \cdot\left((1-t) v_{1}+v_{2}\right) \wedge\left(t v_{1}\right)\right) \\
& =-t(1-t) v_{1} \wedge v_{2}=t^{2} \cdot v_{1} \wedge v_{2}-t \cdot v_{1} \wedge v_{2} .
\end{aligned}
$$

This was the required result.

As remarked in [8], since the specialization $t=1$ gives a representation of the complex group algera $\mathbb{C} \Sigma_{n}$ which is semisimple, all values of $t$ sufficently close to 1 also give a semisimple group algebra. It follows that the representations which we consider are completely reducible. Combining this with the above theorem has the following corollary.

Corollary 2.8. If $V$ is an irreducible submodule in the representation

$$
\rho_{t}: B_{n} \rightarrow \mathrm{GL}\left(\mathbb{C} \Lambda^{p}(M)_{\alpha(t)}\right),
$$

then it is isomorphic to a summand in the Jones representation.

Proof. This is immediate; by Theorem 2.7, there is a map from $H(n, t)$ given by $\pi:\left.g_{i} \rightarrow \rho_{t}\left(\sigma_{i}\right)\right|_{V}$. The Hecke algebra is semisimple, so that $V$ irreducible gives the map $\pi$ an isomorphism on some summand.

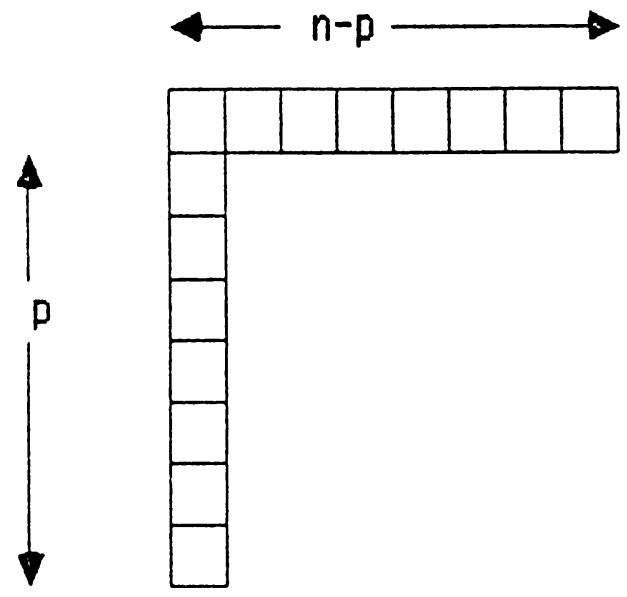

\section{Diagram 1}

The result of Theorem 2.7 is false for the symmetric forms; for if the generators satisfy $(*)$, then the only possibilities for the eigenvalues are 1 and $-t$. But on the symmetric algebra, there is an eigenvalue $t^{2}$ corresponding to the tensor square of a $-t$ eigenvector with itself. In the same vein, the representation on the subspace spanned by vectors of the form $r_{i} \wedge v_{j}$ (where $r_{i}$ is a "real" vector 
in $\mathfrak{S}$ ) is a wreath product of the braid group with the symmetric group. Such representations are rarely quadratic and will not come from the Hecke algebra.

We shall now show that this generalization provides a certain subclass of the Jones representations of $B_{n}$. Our result is in essence equivalent to a result in [8]; in $\S 7$ it is shown that the Hecke algebra representations corresponding to a certain Young diagram come (up to sign) from exterior powers of the Burau representation.

In order to state the result precisely, we need to introduce a notation for a special kind of Young diagram. Recall that in [8] it is shown that there is one representation of $B_{n}$ for each Young diagram of size $n$. Diagram 1 will be denoted $\left(n-p, 1^{p}\right)$.

Theorem 2.9. The representation $\rho_{t}: B_{n} \rightarrow \mathrm{GL}\left(\mathbb{C} \Lambda^{p}(M)_{\alpha(t)}\right)$ contains an irreducible summand of the Jones representation of type $\left(n-p, 1^{p}\right)$.

Proof. As we remarked above, for generic $t$ the representation is semisimple, so completely reducible. By Theorem 2.7 , it is quadratic so that, exactly as in [8], it follows that the submodule decomposition at $t=1$ is the same as the submodule decomposition for $t$ near 1 . Hence in order to prove Theorem 2.9 we need only verify that it holds at the $t=1$ representation of the symmetric group.

This is presumably well known to experts in the representation theory of the symmetric group. However, it can be proved fairly directly. To do this it is necessary to recall a little of the theory of how Young diagrams give rise to a representation of the symmetric group. This material is nicely described in [6, $\S 4]$.

Given a partition of $n$, denoted $\mu, s_{1} \geq s_{2} \geq \cdots \geq s_{k}$, this gives rise to a "diagram" by writing $s_{1}$ boxes in the first row, $s_{2}$ in the second and so on. For example, in this notation Diagram 1 does indeed arise from the partition $n-p, 1,1, \ldots, 1$ (which is abbreviated $n-p, 1^{p}$ ).

We then consider all possible ways of inserting $n$ numbers in this diagram; such an object is called a tableau. If we now regard the tableau as having unordered rows, this is a tabloid. We define a formal vector space $M^{\mu}$ whose generators are the tabloids coming from $\mu$. Then $\Sigma_{n}$ acts on tabloids and hence $M^{\mu}$ in an obvious way; this gives a representation of $\Sigma_{n}$. This representation is not in general irreducible, however it contains an irreducible summand $S^{\mu}$ corresponding to $\mu$; the so-called Specht module.

This is constructed in the following way. Given a tableau, define an element of $M^{\mu}$ by summing all the ways of permuting its columns, attaching to each the sign of the corresponding permutation. This is a polytabloid. The Specht module $S^{\mu}$ is the subspace of $M^{\mu}$ spanned by polytabloids. It follows directly from its definition that the Specht module is an invariant submodule of $M^{\mu}$. 
In fact, one has

Theorem [6, §4]. The ordinary irreducible representations of $\Sigma_{n}$ are exactly the Specht modules $S^{\mu}$.

In our case the situation is particularly simple; we have exactly one column, so that given a tableau $T$, the polytabloid is $\sum_{\sigma \in \Sigma_{p+1}} \operatorname{sign}(\sigma) \cdot \sigma(T)$.

We map a tableau of type $\left(n-p, 1^{p}\right)$, whose column contains the numbers $i_{1}, i_{2}, \ldots, i_{p}$ to the element $v_{i_{1}} \otimes v_{i_{2}} \otimes \cdots \otimes v_{i_{p}}$ in $V \otimes V \otimes \cdots \otimes V$. Recalling that an alternating $p$-tensor is defined by the formula $\sum_{\sigma \in \Sigma_{p}} \operatorname{sign}(\sigma) \cdot \sigma(v)$, we see that each polytabloid is a linear combination of alternating $p$-tensors, so that the $\left(n-p, 1^{p}\right)$-Specht module is carried into $\mathbb{C} \Lambda^{p}(R)_{\alpha(t)}$ by the above map. Since the actions of $\Sigma_{n}$ and $B_{n}$ also correspond, the result follows.

It follows that at least on some summands of the Jones representation there is in some sense no more information than was contained in the Burau representation; in particular from the point of view of faithfulness.

\section{INVARIANT FOLIATIONS AND FORMS}

The diffeomorphsims occurring in the image of the braid and pure braid groups are not purely arbitrary; indeed, we have already observed that they are restricted by the analytic property of Corollary 2.2. In this section we collect further properties and define extra structure on these diffeomorphisms. Throughout this section, we restrict to the case that $G=\mathrm{SU}(2, \mathbb{C})$.

These restrictions will be of two types: the first is the existence of certain invariant (singular) foliations; the second is the existence of an invariant symmetric 2-form. The latter was first observed in the case of the braid group in [15] by Squier. We also show that one of the conjectures of [15] is true.

We first turn our attention to the existence of invariant foliations. Consider the smooth map $\xi: R \rightarrow[-2,2]^{n}$ given by

$$
\left(X_{1}, \ldots, X_{n}\right) \rightarrow\left(\operatorname{tr}\left(X_{1}\right), \ldots, \operatorname{tr}\left(X_{n}\right)\right) .
$$

It is easy to see that the critical values of this map are the points with a coordinate \pm 2 . Given some vector $p \in(-2,2)^{n}$ if we define $M(p)$ to be $\xi^{-1}(p)$, this will be a closed smooth $2 n$-manifold, in fact the result of Lemma 4.3 shows that it is $S^{2} \times S^{2} \times \cdots \times S^{2}$, though we shall not use this in what follows.

We obtain a foliation of $R$ by considering $\left\{M(p) \mid p \in[-2,2]^{n}\right\}$. The generic $M(p)$ is a manifold, but there are singularities coming from critical values. As observed in $\S 2$, the action of the pure braid group preserves each $M(p)$, as trace is a class function. This foliation is also leafwise invariant for the braid group; the difference being that some leaves are permuted by the action of $B_{n}$. The fixed leaves come from those $p$ which have the form $(a, \ldots, a)$. We call such $p$ diagonal. 
This is a strong restriction on the diffeomorphisms occurring in the image of the braid and pure braid groups; in fact, one of the consequences of a result proved below is that the $M(p)$ in some sense carry the Burau and Gassner representations. We can observe this in the following way.

Let $\Gamma$ be the maximal torus of diagonal matrices in $R$. Then for each regular value of $p$, the manifold $M(p)$ meets $\Gamma$ in exactly $2^{n}$ points. This intersection is transverse; one can either see this using a direct geometric argument, since a diagonal matrix in $\mathrm{SU}(2, \mathbb{C})$ is determined locally by its trace, or by appealing to Theorem 3.1 below. Moreover, both manifolds are invariant for the action of the pure braid group. This yields for each $\rho \in \Gamma$ a splitting $T_{\rho}(R)=T_{\rho}(\Gamma) \oplus T_{\rho}(M(p))$. Each factor is $P_{n}$-invariant, indeed the action on the first factor is the identity. If $p$ happens to be diagonal we obtain a $B_{n}{ }^{-}$ invariant splitting, and the action on the first factor is permutational. In fact more is true, and since we shall need it later we record here the following:

Theorem 3.1. Fix some $p \in(-2,2)^{n}$ which is a regular value for $\xi$ and let $\Lambda=\left(\Lambda_{1}, \ldots, \Lambda_{n}\right)$ be one of the diagonal points on $M(p)$. Then $T_{\Lambda}(M(p))$ is spanned by vectors of the form $\left(\Lambda_{1}, \ldots, \mathbf{v} \Lambda_{i}, \ldots, \Lambda_{n}\right)$, where $1 \leq i \leq n$ and $\mathbf{v}$ is a "complex" vector in $\mathfrak{S}$.

Proof. This is a routine calculation. As usual, we identify $T_{\Lambda}(R)$ with $T_{\Lambda_{1}}(\mathrm{SU}(2, \mathbb{C})) \oplus \cdots \oplus T_{\Lambda_{n}}(\mathrm{SU}(2, \mathbb{C}))$. Recall that one has $T_{\Lambda}(M(p))=$ $\operatorname{ker}\left\{d \xi: T_{\Lambda}(R) \rightarrow \mathbf{R}^{n}\right\}$. Considering vectors in the first component for example, a path

$$
v_{t}=\left[\begin{array}{cc}
a_{t} & b_{t} \\
-\bar{b}_{t} & \bar{a}_{t}
\end{array}\right]
$$

gives a tangent vector of the form $\left(v_{t} \Lambda_{1}, \ldots, \Lambda_{n}\right)$. The image of this path is $\lambda_{1} a_{t}+\bar{\lambda}_{1} \bar{a}_{t}+\Sigma\left(\lambda_{i}+\bar{\lambda}_{i}\right)$ and this lies in $\operatorname{ker}(d \xi)$ if and only if

$$
\left.\left(\lambda_{1} \cdot \frac{d}{d t} a_{t}+\bar{\lambda}_{1} \cdot \frac{d}{d t} \bar{a}_{t}\right)\right|_{t=0}=0 .
$$

The condition on the path $v_{t}$ lying in $\mathrm{SU}(2, \mathbb{C})$ is that $a_{t} \bar{a}_{t}+b_{t} \cdot \bar{b}_{t}=1$, so that we also have $\left(\frac{d}{d t} a_{t}+\frac{d}{d t} \bar{a}_{t}\right)_{t=0}=0$; whence since $\lambda_{1} \neq \pm 1$, we have that $\left.\frac{d}{d t} a_{t}\right|_{t=0}=0$ and the path $v_{t}$ gives rise to a complex vector.

Remark. It now follows from the calculations of Theorem 2.4 that the manifolds $M(p)$ carry the interesting part of the Burau and Gassner representations.

We now consider the question of forms. Recall that we decompose $\mathscr{T}^{2}(M)$ as $S^{2}(M) \oplus \Lambda^{2}(M)$, this decomposition being invariant for the natural $B_{n}$ action.

There are 1 -forms along the path $\alpha$ which are left invariant by $B_{n}$; for example the covector associated to the eigenvector at $\alpha(p)$, or the covector which comes from differentiating the map $R \rightarrow \mathbf{R}$ given by $\left(X_{1}, \ldots, X_{n}\right) \rightarrow$ $\Sigma \operatorname{tr}\left(X_{i}\right)$. 
It is obvious to see that there are symmetric 2-forms left invariant by $B_{n}$ in the spaces $S^{2}(M)_{\alpha}$ where $\alpha$ is the usual path. Of course, if $\omega^{1}$ is a $B_{n}$ invariant 1-form, then $\omega^{1} \otimes \omega^{1}$ is invariant, but it is degenerate. We seek forms which are largely nondegenerate along the path $\alpha$.

There is a reasonably natural way to do this; there is a map $h: R \rightarrow \mathbf{R}$ given by

$$
h:\left(X_{1}, \ldots, X_{n}\right) \rightarrow-\operatorname{tr}\left(X_{1} \ldots X_{n}\right) / 2
$$

which is clearly invariant for the action of $B_{n}$ by property (i) of $\S 2$. (We have introduced the factor $-(1 / 2)$ here only to clean up some of the calculation.)

If we restrict to one of the manifolds $M(p)$ with $p$ diagonal, we obtain a function $h: M(p) \rightarrow \mathbb{R}$ and now an easy calculation shows that at (either of) the points $\alpha=(\Lambda, \ldots, \Lambda)$ which lie on $M(p)$, the map $d h_{\alpha}$ is zero on $T_{\alpha}(M(p))$, whence we may form the Hessian of $h$ (see [13]) denoting this by $h_{* *}$. This Hessian defines a symmetric bilinear form

$$
h_{* *}: T_{\alpha}(M(p)) \times T_{\alpha}(M(p)) \rightarrow \mathbf{R}
$$

and will be invariant for the action of the braid group, by construction.

As usual we may perform this operation anywhere along the path $\alpha$ (in the notation of $\S 2)$ where the manifold $M(p)$ is defined; thus we can think of this path of Hessians as a symmetric matrix with entries in one variable.

We shall do some calculations below; it turns out that this Hessian is degenerate. However, we may restrict $h_{* *}$ to a form on the $n-1$ (complex) dimensional subspace of $T_{\alpha}(M(p))$ which is invariant under the action of the braid group. If we let $f_{* *}$ denote the restricted form, this form is clearly still $B_{n}$-invariant. Then we calculate

Theorem 3.2. The Hessian $f_{* *}$ is generically nondegenerate along $\alpha$.

Proof. Again, this is a calculation; to simplify notation, suppose that $n=3$. To compute terms coming from different factors in $T_{\alpha}(M(p))$ we need to compute $\partial^{2} h / \partial s \partial t$ in terms of the form $\left(v_{t} \Lambda, w_{s} \Lambda, \Lambda\right)$ and $\left(v_{t} \Lambda, \Lambda, w_{s} \Lambda\right)$. This yields terms of the shape $-\operatorname{tr}\left(\mathbf{v} \Lambda \mathbf{w} \Lambda^{2}\right) / 2$ and $-\operatorname{tr}\left(\mathbf{v} \Lambda^{2} w \Lambda\right) / 2$, where $\mathbf{v}$ and $w$ are complex vectors.

Let us do a typical case:

$$
\mathbf{v}=\left[\begin{array}{cc}
0 & -1 \\
1 & 0
\end{array}\right], \quad \mathbf{w}=\left[\begin{array}{ll}
0 & i \\
i & 0
\end{array}\right]
$$

One finds that $-\operatorname{tr}\left(\mathbf{v} \Lambda \mathbf{w} \Lambda^{2}\right) / 2=(\lambda i-\bar{\lambda} i) / 2$. If $\lambda=e^{i \theta}$, then this is $-\sin \theta$.

To compute diagonal terms we compute $\partial^{2} h / \partial t^{2}$ of $\left(v_{t} \Lambda, \Lambda, \Lambda\right)$. We shall evaluate this explicitly on the complex basis vectors. Suppose that

$$
v_{t}=\left[\begin{array}{cc}
a_{t} & b_{t} \\
-\bar{b}_{t} & \bar{a}_{t}
\end{array}\right]
$$

is tangent to a complex basis vector. The condition that the path $(v, \Lambda, \Lambda, \Lambda)$ lies in $M(p)$ gives the constraint $\lambda a_{t}+\bar{\lambda} \bar{a}_{t}=\lambda+\bar{\lambda}$ for all $t$. As in Theorem 3.1, we also have the $\mathrm{SU}(2, \mathbb{C})$ condition $a_{t} \bar{a}_{t}+b_{t} \cdot \bar{b}_{t}=1$. 
Then computing $h^{\prime \prime}$ along this path we obtain $h^{\prime \prime}(t)=\lambda^{3} a_{t}^{\prime \prime}+\bar{\lambda}^{3} \cdot \bar{a}_{t}^{\prime \prime}$. We must evaluate $-\frac{1}{2} \cdot h^{\prime \prime}(t)$ at $t=0$.

The constraint yields the equation $\lambda a_{t}^{\prime \prime}+\bar{\lambda} \bar{a}_{t}^{\prime \prime}=0$. Since we were assuming $v_{t}$ tangent to a complex basis vector we have $b_{t}^{\prime}=1$ or $i$ at $t=0$; whence the $\mathrm{SU}(2, \mathbb{C})$ condition gives $a_{t}^{\prime \prime}+\bar{a}_{t}^{\prime \prime}=-2$. From this we deduce the equation

$$
\left.\left(-\frac{1}{2}\right) \cdot h^{\prime \prime}(t)\right|_{t=0}=\left(\lambda^{2}-\bar{\lambda}^{2}\right) /(\bar{\lambda}-\lambda)=-(\lambda+\bar{\lambda})=-2 \cos \theta .
$$

Similar calculations yield the remaining terms. If, as usual, we want to use the complex formalism, it is possible to do this provided that one observes the caveat that since the transpose of the matrix $\left[\begin{array}{c}\cos \theta \sin \theta \\ -\sin \theta \cos \theta\end{array}\right]$ (which we associate to the complex number $\lambda$ ) is a matrix which we associate to the complex number $\bar{\lambda}$, real symmetric forms in the complex formalism should be handled as hermitian matrices. The associated form is then sesquilinear.

One finds that the form $h_{* *}$ has matrix

$$
\left[\begin{array}{ccc}
-(\lambda+\bar{\lambda}) & \bar{\lambda} & \lambda \\
\lambda & -(\lambda+\bar{\lambda}) & \bar{\lambda} \\
\bar{\lambda} & \lambda, & -(\lambda+\bar{\lambda})
\end{array}\right] .
$$

This matrix clearly has eigenvalue zero. To compute $f_{* *}$ we must restrict to the $n-1$ dimensional invariant subspace of $T_{\alpha}(M(p))$. An unenlightening calculation will accomplish this; using as basis the $\lambda^{2}$ eigenvectors for $\sigma_{1}$ and $\sigma_{2} ;$ these are

$$
\left[\begin{array}{c}
\lambda^{2} \\
-1 \\
0
\end{array}\right] \text { and }\left[\begin{array}{c}
0 \\
\lambda^{2} \\
-1
\end{array}\right]
$$

respectively. However it is easier to observe that at $\lambda^{2}=1$, the form becomes $\left[\begin{array}{cc}-6 & 3 \\ 3 & -6\end{array}\right]$ which is clearly nonsingular; so that all nearby values of $\lambda$ give nonsingular matrix; whence the required generic nonsingularity. (Actually, the form is not really defined at $\lambda^{2}=1$; no manifold $M(p)$ is defined, and in the argument above, we divided by $\lambda-\bar{\lambda}$. However a limiting form exists and this suffices.)

The general calculation is similar; one finds that the form is singular, but when restricted to the invariant subspace spanned by $\lambda^{2}$ eigenvectors, at $\lambda^{2}=1$ the form becomes (after a homothety, i.e. multiplication by a matrix of the form $C \cdot$ Id )

$$
\left[\begin{array}{ccccccc}
-2 & 1 & 0 & \ldots & \ldots & \ldots & 0 \\
1 & -2 & 1 & 0 & \ldots & \ldots & 0 \\
0 & 1 & -2 & 1 & 0 & \ldots & 0 \\
\ldots & \ldots & \ldots & \ldots & \ldots & \ldots & \ldots \\
0 & 0 & \ldots & \ldots & 0 & 1 & -2
\end{array}\right]
$$

This proves the result.

Remark. This is the Cartan matrix of the Lie algebra of type $A_{n}$.

Actually it is rather easy to calculate the general form such a symmetric 2tensor must take. We now compute this in the case $n=3$ in a way which is 
easily seen to generalize. For convenience, we work with the unreduced $\mathrm{Bu}-$ rau representation. Denote the obvious basis for $\mathbb{C} T_{\alpha} R$ by $v_{1}, v_{2}, v_{3}$. Then immediately

(i) $\left\langle v_{1}, v_{3}\right\rangle=\left\langle\sigma_{2} v_{1}, \sigma_{2} v_{3}\right\rangle=\left\langle v_{1} \lambda^{2} v_{2}\right\rangle=\bar{\lambda}^{2}\left\langle v_{1}, v_{2}\right\rangle$.

(ii) $\left\langle v_{2}, v_{3}\right\rangle=\left\langle\sigma_{1} v_{2}, \sigma_{1} v_{3}\right\rangle=\left\langle\lambda^{2} v_{1}, v_{3}\right\rangle=\lambda^{2}\left\langle v_{1}, v_{3}\right\rangle$.

(iii) $\left\langle v_{2}, v_{2}\right\rangle=\left\langle\sigma_{1} v_{2}, \sigma_{1} v_{2}\right\rangle=\left\langle\lambda^{2} v_{1}, \lambda^{2} v_{1}\right\rangle=\left\langle v_{1}, v_{1}\right\rangle$.

(iv) $\left\langle v_{3}, v_{3}\right\rangle=\left\langle\sigma_{2} v_{3}, \sigma_{2} v_{3}\right\rangle=\left\langle\lambda^{2} v_{2}, \lambda^{2} v_{2}\right\rangle=\left\langle v_{2}, v_{2}\right\rangle$.

The last constraint is the relationship of the diagonal entries to the offdiagonal entries:

$$
\left\langle v_{1}, v_{1}\right\rangle=\left\langle\sigma_{1} v_{1}, \sigma_{1} v_{1}\right\rangle=\left\langle\left(1-\lambda^{2}\right) v_{1}+v_{2},\left(1-\lambda^{2}\right) v_{1}+v_{2}\right\rangle
$$

which yields (since (iii) and (iv) give $\left\langle v_{1}, v_{1}\right\rangle=\left\langle v_{2}, v_{2}\right\rangle$ )

$$
0=\left(1-\lambda^{2}\right)\left(1-\bar{\lambda}^{2}\right)\left\langle v_{1}, v_{1}\right\rangle+\left\langle v_{2},\left(1-\lambda^{2}\right) v_{1}\right\rangle+\left\langle\left(1-\lambda^{2}\right) v_{1}, v_{2}\right\rangle
$$

or

(v) $\left(1-\lambda^{2}\right)\left(1-\bar{\lambda}^{2}\right)\left\langle v_{1}, v_{1}\right\rangle=\left(\lambda^{2}-1\right)\left\langle v_{1}, v_{2}\right\rangle+\left(\bar{\lambda}^{2}-1\right)\left\langle v_{2}, v_{1}\right\rangle$.

This is the only equation which needs to be satisfied; the other is a consequence of this and (i)-(iv). Since we can suppose that $\lambda \neq \pm 1$, a more symmetric way of writing $(v)$ is

$$
(\bar{\lambda}-\lambda)\left\langle v_{1}, v_{1}\right\rangle=\lambda\left\langle v_{1}, v_{2}\right\rangle-\bar{\lambda}\left\langle v_{2}, v_{1}\right\rangle .
$$

The solution provided by the calculation of Theoem 3.2 is the case $\left\langle v_{1}, v_{2}\right\rangle=\lambda$.

Other solutions to these equations exist; these include for example $\left\langle v_{1}, v_{2}\right\rangle=$ $\bar{\lambda}^{3}$ and $\left\langle v_{1}, v_{1}\right\rangle=\lambda+\bar{\lambda}$. This gives the form

$$
\left[\begin{array}{ccc}
\lambda+\bar{\lambda} & \lambda^{3} & \lambda^{5} \\
\bar{\lambda}^{3} & \lambda+\bar{\lambda} & \lambda^{3} \\
\bar{\lambda}^{5} & \bar{\lambda}^{3} & \lambda+\bar{\lambda}
\end{array}\right] \text {, or, in general, }\left[\begin{array}{ccccc}
\lambda+\bar{\lambda} & \lambda^{3} & \lambda^{5} & \lambda^{7} & \ldots \\
\bar{\lambda}^{3} & \lambda+\bar{\lambda} & \lambda^{3} & \lambda^{5} & \ldots \\
\bar{\lambda}^{5} & \bar{\lambda}^{3} & \lambda+\bar{\lambda} & \lambda^{3} & \ldots \\
\bar{\lambda}^{7} & \bar{\lambda}^{5} & \bar{\lambda}^{3} & \lambda+\bar{\lambda} & \ldots
\end{array}\right] .
$$

Notice that this second form is not degenerate, so that as a form it is not equivalent to $h_{* *}$ (equivalent forms have determinants differing by a nonzero square) but a somewhat tedious calculation shows that when restricted to the irreducible representation our second form differs from the first by homothety. It follows that their isometry groups are the same.

It is also possible to verify that the second form is equivalent to the form of [15] when restricted to the $(n-1)$ dimensional subspace, at least after a change of basis. Our approach has naturality merits; for example it shows where the form came from, and why the substitution $t=s^{2}$ was necessary; the $s$ of [15] is exactly our $\lambda$.

Moreover, our procedure actually shows more; namely that there is an invariant form for the pure braid group: 
Theorem 3.3. There is a symmetric nonsingular matrix with entries in $n$ indeterminates which is preserved (as a form) by the reduced Gassner representation.

Proof. The same procedure applied to $f$ on $M(p)$, where $p$ is not necessarily diagonal, yields invariant Hessians on the whole of the maximal torus. This can be interpreted as a matrix with entries in $n$ indeterminates. Choose some diagonal $p$ where $f_{* *}$ is nondegenerate; then the Hessians in an open neighborhood of this one will be nondegenerate; whence they are nondegenerate generically.

At this stage we interject a short digression. In [15], two conjectures are offered, the truth of both being sufficent to establish the faithfulness of the Burau representation on all the braid groups simultaneously. Let us remark here that if one rephrases these conjectures for a fixed $n$, then the second one becomes true. Specifically, let $\beta^{(k)}$ be the representation obtained from the Burau representation of $B_{n}$ by substituting $\tau_{k}$ for $-t$, where $\tau_{k}$ is a primitive $k$ th root of unity, and let $N_{k}$ be the normal closure of the elements $\sigma_{1}^{k}, \ldots, \sigma_{n-1}^{k}$. Then the conjectures are

(C1) $N_{k}$ is the kernel of $\beta^{(k)}$.

(C2) The intersection of the $N_{k}$ 's is trivial.

Clearly, the truth of this pair of conjectures establishes the faithfulness of the Burau representation for $B_{n}$. However, we have

Theorem 3.4. (C2) is true.

Proof. Suppose that $g$ is a nontrivial element of $B_{n}$ lying in $N_{k}$ for every $k$. Then since $B_{n}$ is residually finite, there is a normal subgroup of finite index, $K \triangleleft B_{n}$, with $g \notin K$. Since $K$ has finite index, there is an $r$ so that $\sigma_{i}^{r}$ lies in $K$ for each $i$, and the fact that $K$ is normal implies that $N_{r} \leq K$. But then $g \in K$, a contradiction.

Since we have remarked that these representations arise via the tangent bundle, it is natural to observe that there is a little extra structure on the cotangent bundle representations.

In order to do this we recall that a manifold $M$ (necessarily even dimensional) has a symplectic structure if there is a closed, nondegenerate 2-form on $M$, which we shall denote by $\omega^{2}$.

Let us recall the definitions of some of these terms in symbols; $\omega^{2}$ is required to have $d \omega^{2}=0$ and for every $x \in M$ and every $v \in T M_{x}$, there is a $w \in T M_{x}$ with $\omega^{2}(v, w) \neq 0$. We say the pair $\left(M, \omega^{2}\right)$ is a symplectic manifold.

In our context, we shall deal with the cotangent bundle of $R$, which we denote by $T^{*} R$. The points in this bundle are of the form $\left(\rho, \nu_{\rho}\right)$, where $\rho$ is a representation in $R$, and $\nu_{\rho}$ is a covector based at $\rho$, that is, a linear map $\nu_{\rho}: T(R)_{\rho} \rightarrow K$. 
A diffeomorphism $\theta: R \rightarrow R$ defines a diffeomorphism denoted by (to avoid proliferation of notation) $\Theta: T^{*} R \rightarrow T^{*} R$ given by

$$
\Theta\left(\rho, \nu_{\rho}\right)=\left(\theta \rho, \nu_{\rho} \circ(d \theta)^{-1}\right) \text {. }
$$

It is rather easily checked that this is a covariant assignment. (Notice that we have substituted $\theta^{-1}$ for $\theta$ of the usual definition for exactly this reason.) We shall use this action for the braid and pure braid groups.

The cotangent bundle of any smooth manifold admits a canonical symplectic structure (we shall indicate how below) and we have

Theorem 3.5. The action of $B_{n}$ and $P_{n}$ on $T^{*} R$ both preserve the canonical symplectic structure.

Remark. In some sense this is selfevident given the use of the word "canonical"; however, since our point of view is that of a low-dimensional topologist, proving it is an excuse to define some of the terms. General facts concerning symplectic manifolds are contained in [16], for example.

Proof. To define the canonical 2-form, we first define a preferred 1-form $\omega^{1}$ on $T^{*} R$. So suppose that $\xi$ is a tangent vector to $T^{*} R$ running through the point $(\rho, \nu)$, that is it lies in $T_{(\rho, \nu)}\left(T^{*} R\right)$.

Notice that we have a map $\pi: T^{*} R \rightarrow R$, which we can differentiate to get a map $d \pi: T_{(\rho, \nu)}\left(T^{*} R\right) \rightarrow T R_{\rho}$. We define the $1-$ form $\omega^{1}$ by the rule $\omega^{1}(\xi)=\nu(d \pi(\xi))$.

Then we define $\omega^{2}=d \omega^{1}$. This is clearly closed, and can be shown to be nondegenerate. (See [16] for example.)

It now follows that if $\theta: R \rightarrow R$ is any diffeomorphism, if we lift to $\Theta: T^{*} R \rightarrow T^{*} R$ in the manner described above, then $\Theta^{*} \omega^{1}=\omega^{1}$. For if $\xi \in T_{(\rho, \nu)}\left(T^{*} R\right)$, then the commutative diagram

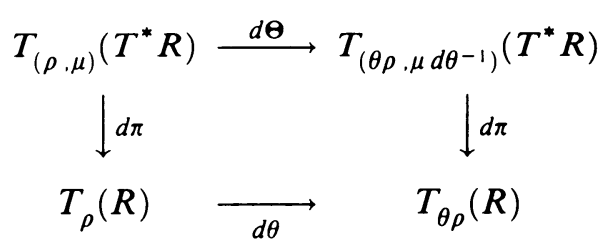

gives that $\boldsymbol{\Theta}^{*} \omega^{1}(\xi)=\omega^{1}(d \Theta(\xi))=\nu \circ(d \theta)^{-1}(d \pi(d \Theta(\xi)))=\nu \circ(d \theta)^{-1} d \theta(d \pi(\xi))$ and the right-hand side is precisely $\omega^{1}(\xi)$.

Hence $\boldsymbol{\Theta}^{*} \omega^{2}=\Theta^{*} d \omega^{1}=d\left(\Theta^{*} \omega^{1}\right)=\omega^{2}$, as was required.

In this way we see that if we denote the point $\left(\Lambda_{p}, \ldots, \Lambda_{p}\right)$ by $\alpha(p)$, then we can lift into the cotangent bundle and consider the action of the braid group on the points of the form $(\alpha(p), \nu)$.

Notice that we have already observed that the braid group has fixed vectors in $T_{\alpha(p)}(R)$ for every $p$; hence there are fixed covectors, which we can choose 
continuously (but not canonically) to give a path of fixed points $\left(\alpha(p), \nu_{p}\right)$ in $T^{*} R$.

Exactly as usual we get linear representations of the braid group by linearizing along the path $\alpha^{*}(p)=\left(\alpha(p), \nu_{p}\right)$; the map is $\theta \rightarrow d \Theta$. Then the above theorem implies that the action of the braid group fixes the canonical 2-form when it is restricted to this path. Thus we have shown:

Theorem 3.6. The representation of $B_{n}$ given by $\theta \rightarrow d \Theta_{\alpha^{*}(p)}$ fixes the canonical symplectic 2-form $\left.\omega^{2}\right|_{\alpha^{*}(p)}$.

It is easy to see that this cotangent bundle representation contains the Burau representation in the following way: there is a diffeomorphism $R \times \mathfrak{S}^{*} \rightarrow T^{*}(R)$ (where $\mathfrak{S}^{*}$ is the covectors based at the identity) given by mapping $(\rho, \nu)$ to $\left(\rho, \nu \circ\left(d r_{\rho}\right)^{-1}\right)$, where $r_{\rho}: R \rightarrow R$ is right multiplication by $\rho$. Then the action of $\Theta$ on $T^{*}(R)$ is conjugated to an action on $R \times \mathfrak{S}^{*}$ given by

$$
(\rho, \nu) \rightarrow\left(\theta \rho, \nu \circ\left(d r_{\rho}\right)^{-1} \circ\left(d \theta_{\rho}\right)^{-1} \circ d r_{\theta \rho}\right)
$$

In this way we see that in the particular case that $\nu_{p}$ is a nonzero covector for all $p$ fixed by all of $B_{n}$ (that is, the path we consider is $\alpha^{*}(p)=\left(\alpha(p), \nu_{p}\right)$ ), we can identify $T_{\alpha(p), \nu}\left(T^{*} R\right)$ with $T_{\alpha(p)}(R) \times \mathfrak{S}^{*}$ where the first factor is invariant for the $B_{n}$ action.

Of course, we also have

Theorem 3.7. The representation of $P_{n}$ given by $\theta \rightarrow d \boldsymbol{\Theta}_{\beta^{*}}$ fuxes the canonical symplectic 2-form $\left.\omega^{2}\right|_{\beta^{*}}$.

\section{EXAMPLES}

The first nontrivial example of Theorem 2.9 is the $3,1^{2}$ representation of $B_{5}$. Use of the hook rule shows that this should be an irreducible representation of dimension 6. In this case the unreduced Burau acts on $T_{\alpha}(R)$ which has complex dimension 5 and there is an invariant subspace of dimension 4 which is the reduced Burau representation. Denoting this space by $V$, we see that $\Lambda^{2}(V)$ has dimension 6 . A computation reveals that the representation is given by:

$$
\sigma_{1} \rightarrow\left[\begin{array}{cccccc}
-t & 0 & 0 & 1 & 0 & 0 \\
0 & -t & 0 & 0 & 1 & 0 \\
0 & 0 & -t & 0 & 0 & 0 \\
0 & 0 & 0 & 1 & 0 & 0 \\
0 & 0 & 0 & 0 & 1 & 0 \\
0 & 0 & 0 & 0 & 0 & 1
\end{array}\right], \quad \sigma_{2} \rightarrow\left[\begin{array}{cccccc}
1 & 0 & 0 & 0 & 0 & 0 \\
0 & 1 & 0 & 0 & 0 & 0 \\
0 & 1 & -t & 0 & 0 & 0 \\
t & 0 & 0 & -t & 0 & 1 \\
0 & t & 0 & 0 & -t & 0 \\
0 & 0 & 0 & 0 & 0 & 1
\end{array}\right]
$$




$$
\sigma_{3} \rightarrow\left[\begin{array}{cccccc}
1 & 0 & 0 & 0 & 0 & 0 \\
1 & -t & t & 0 & 0 & 0 \\
0 & 0 & 1 & 0 & 0 & 0 \\
0 & 0 & 0 & 1 & 0 & 0 \\
0 & 0 & 0 & 1 & -t & 0 \\
0 & 0 & 0 & t & 0 & -t
\end{array}\right], \quad \sigma_{4} \rightarrow\left[\begin{array}{cccccc}
-t & t & 0 & 0 & 0 & 0 \\
0 & 1 & 0 & 0 & 0 & 0 \\
0 & 0 & 1 & 0 & 0 & 0 \\
0 & 0 & 0 & -t & t & 0 \\
0 & 0 & 0 & 0 & 1 & 0 \\
0 & 0 & 0 & 0 & 0 & -t
\end{array}\right] .
$$

Direct calculation shows that this is a representation, and the reader can verify that the quadratic relation $g^{2}=(1-t) g+t$ holds for the generators. Moreover, a character table computation shows that setting $t=1$ yields the irreducible 6 dimensional representation of $\Sigma_{5}$.

As a second example, let us observe that we can apply some results in the theory of Lie groups to produce other representations in a very natural way. To do this we use the groups $\mathrm{SU}(k+1, \mathbb{C})$. Recall that the maximal torus $T$ in these groups consists of the diagonal matrices $\left(\lambda_{1}, \lambda_{2}, \ldots, \lambda_{k}, \Pi \bar{\lambda}_{i}\right)$, where each $\lambda_{i}$ lies on the unit circle in $\mathbb{C}$. If we let the normalizer of $T$ in $\mathrm{SU}(k+1, \mathbb{C})$ be $N(T)$, then recall that the Weyl group, which is by definition $W=N(T) / T$ acts on this torus. In the case $\mathrm{SU}(k+1, \mathbb{C}), W$ is the symmetric group on $k+1$ letters, which we denote $\Sigma(k+1)$. Generators in this case can be easily seen; they are matrices of the form

$$
\left[\begin{array}{ccccc}
\mathrm{Id} & 0 & \ldots & \ldots & 0 \\
& 0 & 1 & \ldots & 0 \\
& -1 & 0 & \ldots & 0 \\
& & & & \text { Id }
\end{array}\right]=M(i, i+1)
$$

where the 1 is the $(i, i+1)$ entry. Of course this is not a splitting of the Weyl group into $\mathrm{SU}(k+1, \mathbb{C})$; each $M(i, i+1)$ has order four. However we do have

Lemma 4.1.

$$
\begin{aligned}
& M(i, i+1) M(i+1, i+2) M(i, i+1) \\
& \quad=M(i+1, i+2) M(i, i+1) M(i+1, i+2) .
\end{aligned}
$$

(b) $\quad M(i, i+1) M(j, j+1)=M(i, i+1) M(j, j+1) \quad$ for $|i-j|>1$.

Proof. Routing checking.

So we define as usual $\operatorname{Ad}(i, i+1)$ to be the diffeomorphism of $\mathrm{SU}(k+1, \mathbb{C})$ defined by conjugation by $M(i, i+1)$. Then we have the analogue of the above lemma, where Ad replaces $M$, though the Ad maps still have order 4. Set $R=R\left(F_{k}, \mathrm{SU}(k+1, \mathbb{C})\right)$, and as usual extend the action of Ad by simultaneous action in each factor. Then we obtain

Theorem 4.2. The map $\sigma_{i} \rightarrow \operatorname{Ad}(i, i+1) \circ \sigma_{i}^{*}$ defines a faithful representation $B_{k} \rightarrow \operatorname{Diff}(R)$.

Proof. As observed in $\S 2$, any associated map commutes with an Ad map, so the fact that the map is a representation follows directly from the Ad version of Lemma 4.1 . 
Notice that this representation coincides with that of Theorem 2.4 on a normal subgroup of finite index in $B_{k}$. Thus the representation is faithful on a normal subgroup. The fact that the whole representation is faithful now follows from [9].

Remark. Notice that this does not use the whole Weyl group of $\mathrm{SU}(k+1, \mathbb{C})$.

We can use this more complicated representation to obtain a linear representation as in Theorem 2.3. We may choose a path $\alpha(p)=$ diagonal matrices $\left(\lambda_{p}, \lambda_{p}, \ldots, \lambda_{p},\left(\lambda_{p}\right)^{k}\right)$. (It is at this point we must use only the initial $k$ segment $\Sigma(k) \leq W$, in order to get a noncentral fixed path.) Then exactly as above we obtain a one-parameter family of representations

$$
\rho_{p}: B_{k} \rightarrow \operatorname{Diff}(R) \rightarrow \mathrm{GL}\left(T_{\alpha(p)}(R)\right)
$$

defined by $\sigma_{i} \rightarrow d\left(\operatorname{Ad}(i, i+1) \circ \sigma_{i}^{*}\right)_{\alpha(p)}$. In fact it is easy to see that this representation comes from a wreath product; these representations are rarely quadratic, and so will not arise from the Hecke algebra.

As a final example, let us briefly observe that we can pursue this idea in a somewhat different direction to obtain interesting representations by diffeomorphisms of other naturally occurring groups.

Consider the group $\operatorname{Aut}\left(F_{2}\right)$; the automorphism group of the free group of rank two. It is well known that when this group acts on $F_{2}$ it preserves the commutator $[x, y]$ up to conjugacy and inversion. (See the book by Magnus, Karrass and Solitar, or check it for yourself by using the Nielsen generators.) If $M, N$ are matrices in $\mathrm{SU}(2, \mathbb{C})$, then $M$ and $N$ differ by conjugacy and inversion if and only if $\operatorname{tr}(M)=\operatorname{tr}(N)$. This is the basis of the following construction. We need the following lemma.

Lemma 4.3. Let $\operatorname{tr}: \mathrm{SU}(2, \mathbb{C}) \rightarrow[-2,2]$ be the trace map. Then this is a smooth map and the only critical points are $\pm \mathrm{Id}$. If $-2<p<2$, then $\operatorname{tr}^{-1}(p)$ is a smooth 2-sphere.

Proof. The map tr is given by real polynomials, so it is smooth. It is easy to see that any matrix of trace other than \pm 2 is noncritical by diagonalizing for example.

Observe that

$$
\operatorname{tr}^{-1}(p)=\left[\begin{array}{cc}
a & b \\
-\bar{b} & \bar{a}
\end{array}\right], \quad a+\bar{a}=p .
$$

Considering this as a real algebraic variety, this can be written as the solution set of the equations

$$
a_{1}^{2}+a_{2}^{2}+b_{1}^{2}+b_{2}^{2}=1, \quad 2 a_{1}=p,
$$

where $a=a_{1}+i a_{2}$, etc. This is the same as the single equation

$$
a_{2}^{2}+b_{1}^{2}+b_{2}^{2}=1-(p / 2)^{2} \text {. }
$$

Since $|p|<2$, the constant on the right-hand side is positive, so the solutions form a 2-sphere. 
Let $S_{p}$ be the set $\operatorname{tr}^{-1}(p)$; implicitly we shall always assume that $-2<p<$ 2. There is a map $\partial: \operatorname{SU}(2, \mathbb{C}) \times \mathrm{SU}(2, \mathbb{C}) \rightarrow \mathrm{SU}(2, \mathbb{C})$ given by $X, Y \rightarrow$ $[X, Y]$. It is well known that the critical points of this map are exactly the reducible representations.

For every $y \in S_{p}$, this implies that any $(a, b) \in \partial^{-1}(y)$ is a regular point for $\partial$, so that the map $\partial$ is everywhere transverse to $S_{p}$. It follows that $\partial^{-1}\left(S_{p}\right)$ is a closed, smooth 5-manifold in $\mathrm{SU}(2, \mathbb{C}) \times \mathrm{SU}(2, \mathbb{C})$. Call this manifold $N_{p}$.

Lemma 4.4. $\bigcup\left\{N_{p} \mid p \in(-2,2)\right\}$ is diffeomorphic to a product $N_{0} \times(-2,2)$.

Proof. There is a natural smooth projection tro $\partial: \bigcup\left\{N_{p} \mid p \in(-2,2)\right\} \rightarrow$ $(-2,2)$ given by $N_{p} \rightarrow p$.

Observe that $\bigcup\left\{N_{p} \mid p \in(-2,2)\right\}$ is a closed smooth 6-manifold inside $\mathrm{SU}(2, \mathbb{C})^{2}$. If $x$ is a point in the domain lying in level $q$, say, then the map

$$
d \partial_{x}: T_{x}\left(\bigcup\left\{N_{p} \mid p \in(-2,2)\right\}\right) \rightarrow T \partial_{x}(\mathrm{SU}(2, \mathbb{C}))
$$

is a surjection. As we observed above, the map

$$
d \operatorname{tr}_{y}: T_{y}(\mathrm{SU}(2, \mathbb{C})) \rightarrow T_{\operatorname{tr}(y)}(-2,2)
$$

is a surjection for every noncentral $y$, so that $x$ is a regular point for $\operatorname{tr} \circ \partial$. Hence this Morse function has no critical points, so by [13] the required result follows.

In particular, if $\theta$ is any element of $\operatorname{Aut}\left(F_{2}\right)$, then it has an associated map which induces a diffeomorphism of each of the manifolds $N_{p}$ by our initial observation concerning the commutator. The induced map of the product $N_{0} \times(-2,2)$ is level preserving and smooth.

We can make this into a representation of $\operatorname{Aut}\left(F_{2}\right)$ as a group of diffeomorphisms via the associated map construction and we obtain a homomorphism $\operatorname{Aut}\left(F_{2}\right) \rightarrow \operatorname{Diff}\left(N_{p}\right)$. Using the product structure we obtain a parametrized family of representations $\rho_{p}: \operatorname{Aut}\left(F_{2}\right) \rightarrow \operatorname{Diff}\left(N_{0}\right)$.

As in Theorem 2.1, we have the following consequene.

Lemma 4.5. The representation $\rho_{p}: \operatorname{Aut}\left(F_{2}\right) \rightarrow \operatorname{Diff}\left(N_{0}\right)$ is faithful.

Proof. By using [5], we see that we may choose a pair of matrices $X, Y$ in $\mathrm{SU}(2, \mathbb{C})$, so that the group that $X$ and $Y$ generate is a free group on two generators, so that in particular their commutator is not - Id. Suppose that $X, Y$ lie in $N_{p}$. Exactly as usual it now follows that if $\theta$ is not the identity automorphsim, the associated map is not the identity on $N_{p}$.

We now examine the manifold $N_{0}$. Notice that the conjugation action of $\mathrm{SU}(2, \mathbb{C})$ on itself gives rise to a free $\mathrm{SO}(3)$ action on $N_{0}$. Our first result concerns this action.

Lemma 4.6. $N_{0} / \mathrm{SO}(3)=S^{2}$.

Proof. Recall that $N_{0}=\{(X, Y) \mid \operatorname{tr}[X, Y]=0\}$. In the $\mathrm{SO}(3)$ quotient space we may assume that $X$ is diagonal, and by a further conjugacy if necessary 
suppose that $X=\left[\begin{array}{ll}\lambda & 0 \\ 0 & \bar{\lambda}\end{array}\right]$ with $0 \leq \arg (\lambda) \leq \pi$. If the matrix $Y$ is moved to $\left[\begin{array}{cc}c & d \\ -d & \bar{c}\end{array}\right]$ then as above we arrange that $d$ is real and strictly positive ( $d$ cannot be zero here). The constraint is then $2|c|^{2}+\lambda^{2}|d|^{2}+\bar{\lambda}^{2}|d|^{2}=0$. Writing $|c|^{2}+d^{2}=1$, and $\lambda=e^{i \theta}$, we deduce the equation $d^{2} \sin ^{2} \theta=1 / 2$.

If $d=1$, then there are precisely two solutions for $\theta$ in the range, and exactly one value for $c$, namely zero. For $1>d>1 / \sqrt{2}$, there continue to be two solutions in $\theta$, but now each gives a circle of solutions in $c$. These two circles approach each other until at $d^{2}=1 / 2$ there is exactly one solution for $\theta$. In this way we see that $N_{0} / \mathrm{SO}(3)$ is a 2 -sphere as required.

This gives an action of $\operatorname{Aut}\left(F_{2}\right)$ on $S^{2}$; the difficulty with this action is that it cannot be faithful, since the action of inner automorphisms is clearly lost (though as we show below, no more than this is lost).

However there is in fact a slightly smaller manifold which it is useful to consider in the context of $\operatorname{Aut}\left(F_{2}\right)$; this arises by restriction of the $\mathrm{SO}(3)$ action to the diagonal subgroup. This action is denoted $\operatorname{Ad}\left(S^{1}\right)$ as in $\S 2$. We define $\bar{N}$ to be $N_{0} / \operatorname{Ad}\left(S^{1}\right)$. This is a closed smooth 4-manifold. The utility of this manifold is containd in the following:

Theorem 4.7. (a) $\operatorname{Out}\left(F_{2}\right)$ acts faithfully on $S^{2}$.

(b) $\operatorname{Aut}\left(F_{2}\right)$ acts faithfully on $\bar{N}$.

Proof. (a) A result of Nielsen implies that $\operatorname{Out}\left(F_{2}\right)=\mathrm{GL}_{2}(\mathbb{Z})$. It follows rather easily that if $\theta$ represents a nontrivial element of $\operatorname{Out}\left(F_{2}\right)$ then we can find a commuting pair of matrices $X$ and $Y$ with $\operatorname{tr}(X) \neq \operatorname{tr}(\Theta(X))$ or $\operatorname{tr}(Y) \neq$ $\operatorname{tr}(\Theta(Y))$, say the former. Then by perturbing $Y$ very slightly, we can arrange a pair $(X, Y)$, with $\operatorname{tr}(X) \neq \operatorname{tr}(\Theta(X))$ and $\operatorname{tr}[X, Y]=p \neq \pm 2$. The $\operatorname{SO}(3)$ class of this element in $N_{p}$ gives a point not fixed by the $\Theta$-action. The result follows.

(b) Since we have a map $\bar{N}=N / \operatorname{Ad}\left(S^{1}\right) \rightarrow N / \operatorname{SO}(3)$, the result of (a) implies that any element in the kernel of the map $\operatorname{Aut}\left(F_{2}\right) \rightarrow \operatorname{Diff}(\bar{N})$ must be an inner automorphism. Extending Epstein's result slightly, it is easy to see that we can choose a pair of matrices with $X, Y$ generating a free group of rank 2 containing no nontrivial diagonal matrices. The claim is that this pair represents a point in $\bar{N}$ moved by every inner automorphism. For if not, then there is a diagonal $\Lambda$ with $\Lambda X \Lambda^{-1}=\Theta(X)=M X M^{-1}$ and $\Lambda(Y)=M Y M^{-1}$, where $M$ is a nontrivial element of the group generated by $X$ and $Y$, coming from the inner automorphism. Then $\Lambda^{-1} \cdot M$ centralizes both $X$ and $Y$, so is central, implying that $M$ is diagonal, a contradiction.

The manifold $\bar{N}$ seems to be the smallest manifold on which there is hope for the representation to be faithful, so it is this manifold upon which we shall focus. We first note that the sequence $\mathrm{SO}(3) \rightarrow N \rightarrow S^{2}$ descends to a sequence 
$\operatorname{SO}(3) / \operatorname{Ad}\left(S^{1}\right) \rightarrow \bar{N} \rightarrow S^{2}$, since the $\operatorname{Ad}\left(S^{1}\right)$ conjugation action is the identity over the base. Also, $\operatorname{SO}(3) / \operatorname{Ad}\left(S^{1}\right)$ is easily seen to be $S^{2}$, as the Hopf sequence $S^{1} \rightarrow S^{3} \rightarrow S^{2}$ factors through $S^{1} \rightarrow S^{3} \rightarrow \mathrm{SO}(3) \rightarrow S^{2}$.

The sequence $S^{2} \rightarrow \bar{N} \rightarrow S^{2}$ is a fibration; so that $\bar{N}$ is simply connected. Moreover, applying the Gysin sequence we see that $H_{2}(\bar{N}) \doteq \mathbb{Z} \oplus \mathbb{Z}$.

In fact we can do rather better than this; there is a pair of $S^{2}$ 's inside $\bar{N}$ which we can identify. Let $G_{1}=\left\{(X, Y) \mid[X, Y]=\Lambda_{i}\right\} / \operatorname{Ad}\left(S^{1}\right)$ and $G_{2}=$ $\left\{\left(\Lambda_{\lambda}, Y\right) \mid \arg (\lambda)>0\right.$ and $\left.\operatorname{tr}\left[\Lambda_{\lambda}, Y\right]=0\right\} / \operatorname{Ad}\left(S^{1}\right)$. It is easy to verify directly (or see [10]) that these are both spheres in $\bar{N}$, and they clearly meet in one point. Using this we can show

Theorem 4.8. $\operatorname{Aut}\left(F_{2}\right)$ acts on $H_{2}(\bar{N})$ as a finite groups.

Proof. The results of Nielsen imply that $\operatorname{Aut}\left(F_{2}\right)$ is generated by the three automorphisms:

$$
D: \begin{gathered}
x \rightarrow x \\
y \rightarrow y x
\end{gathered}, \quad F: \begin{gathered}
x \rightarrow y \\
y \rightarrow x
\end{gathered}, \quad I: \begin{gathered}
x \rightarrow x^{-1} \\
y \rightarrow y
\end{gathered} .
$$

Thus it suffices to check the action on these. The action of $D$ on $G_{1}$ is to fix it, up to orientation, since $D$ preserves the commutator. $G_{2}$ is also clearly fixed, up to orientation. So $D$ maps to $\left[\begin{array}{ll}\alpha & 0 \\ 0 & \beta\end{array}\right]$ where $\alpha, \beta \in( \pm 1)$.

Similarly, the flip inverts the commutator, so that $G_{1}$ is carried to $G_{1}$ (which is the sphere analogous to $G_{1}$, but with $\Lambda_{-i}$ ). Since $G_{1}^{\prime}$ is homologous to $G_{1}$ (it meets $G_{2}$ in one point and is disjoint from $G_{1}$ ) we see that $F$ maps to $\left[\begin{array}{ll}\alpha & 0 \\ 0 & \beta\end{array}\right]$ and has order 2 .

Inversion maps $G_{2}$ to $G_{2}^{\prime}$ (defined by $\arg \lambda<0$ ) so has matrix $\left[\begin{array}{cc}\alpha & 0 \\ 0 & \beta\end{array}\right]$. These matrices generate a finite group in every case.

This action will be explored at greater length in a subsequent paper.

\section{REFERENCES}

1. J. Frank Adams, Lectures on Lie groups, Univ. of Chicago Press, 1969.

2. J. Birman, Braids, links and mapping class groups, Ann. of Math. Studies, no. 82, Princeton Univ. Press, Princeton, N.J., 1975.

3. __ On the Jones polynomial of closed 3-braids, Invent. Math. 81 (1985), 287-294.

4. W. Burau, Uber Zopfgruppen und gleichsinnig verfrillte Verkettunger, Abh. Math. Sem. Hanischen Univ. 11 (1936), 171-178.

5. D. B. A. Epstein, Almost all subgroups of a Lie group are free, J. Algebra 19 (1971), 261-262.

6. G. D. James, Representation theory of the symmetric groups, Lecture Notes in Math., vol. 682, Springer-Verlag, Berlin and New York.

7. V. F. R. Jones, A polynomial invariant for knots via von Neuamnn algebras, Bull. Amer. Math. Soc. (N.S.) 12 (1985), 103-111.

8. 126 (1987), 335-388.

9. D. D. Long, A note on the normal subgroups of mapping class groups, Math. Proc. Cambridge Philos. Soc. 99 (1986), 79-87. 
10. __ Representations of fibred 3-manifold groups into a compact Lie group, preprint.

11. W. Magnus, Rings of Fricke characters and automorphism groups of free groups, Math. Z. 170 (1980), 91-103.

12. J. W. Milnor, Characteristic classes, Ann. of Math. Studies, no. 76, Princeton Univ. Press, Princeton, N.J., 1974.

13. __ Morse theory, Ann. of Math. Studies, no. 51, Princeton Univ. Press, Princeton, N.J., 1963.

14. M. Spivak, A comprehesive introduction to differential geometry, Publish or Perish.

15. C. Squier, The Burau representation is unitary, Proc. Amer. Math. Soc. 90 (1984), 199-202.

16. A. Weinstein, Lectures on symplectic manifolds, CBMS Regional Conf. Ser. Math., no. 29, Amer. Math. Soc., Providence, R.I., 1977; reprinted 1983.

Department of Mathematics, University of California, Santa Barbara, California 93106 